International Mathematical Forum, Vol. 9, 2014, no. 30, 1447 - 1450

HIKARI Ltd, www.m-hikari.com

http://dx.doi.org/10.12988/imf.2014.48153

\title{
Some Limits of Sequences of Euler Mascheroni Kind
}

\author{
Daniele Ritelli \\ University of Bologna \\ Department of Statistics \\ Via Belle Arti 41 \\ 40126 Bologna Italy \\ Calogero Salvatore Siracusa \\ Via Roma, 132 \\ 92010 Siculiana (AG) Italy
}

Copyright (c) 2014 Daniele Ritelli and Calogero Salvatore Siracusa. This is an open access article distributed under the Creative Commons Attribution License, which permits unrestricted use, distribution, and reproduction in any medium, provided the original work is properly cited.

\begin{abstract}
In this short note we evaluate some limits of sequences related to the Euler Mascheroni $\gamma$ constant

$$
\gamma=\lim _{n \rightarrow \infty}\left(\sum_{m=1}^{n} \frac{1}{m}-\ln n\right)
$$
\end{abstract}

Mathematics Subject Classification: 40A05, 11B83

Keywords: Euler Mascheroni $\gamma$, Zeta Function

\section{Introduction}

We study some sequences $\left(x_{n}\right)$ with general term

$$
x_{n}=a_{n}-b_{n}
$$


where the sequences $\left(a_{n}\right)$ and $\left(b_{n}\right)$ are positively divergent and equivalent, i.e.:

$$
\lim _{n \rightarrow \infty} a_{n}=\lim _{n \rightarrow \infty} b_{n}=+\infty, \quad \lim _{n \rightarrow \infty} \frac{a_{n}}{b_{n}}=1 .
$$

There are, see [3] Theorem 1 therein, conditions which enable the computation of the limit

$$
\lim _{n \rightarrow \infty} x_{n}=\lim _{n \rightarrow \infty}\left(a_{n}-b_{n}\right)
$$

such conditions are related to the growth of the sequence $\left(b_{n}\right)$ and the ratio $a_{n} / b_{n}$, namely if there exist positive numbers $p, q$ such that

$$
\lim _{n \rightarrow \infty} \frac{b_{n}}{n^{p}}=b>0, \quad \lim _{n \rightarrow \infty}\left(\frac{a_{n}}{b_{n}}\right)^{n^{q}}=a>0
$$

then

$$
\lim _{n \rightarrow \infty}\left(a_{n}-b_{n}\right)= \begin{cases}0 & \text { if } p<q \\ b \ln a & \text { if } p=q \\ +\infty & \text { if } p>q, a>1 \\ -\infty & \text { if } p>q, a<1\end{cases}
$$

This result is useful in order to compute the limit of the famous Lalescu sequence

$$
\lim _{n \rightarrow \infty}(\sqrt[n+1]{(n+1) !}-\sqrt[n]{n !})=\frac{1}{e}
$$

proposed by the Romanian Mathematician T. Lalescu in 1900.

Observe that the most intriguing and famous sequence of the form (1) is the sequence which give the $\gamma$ constant, see [1] for a exhaustive treatment of this important mathematical constant,

$$
\gamma=\lim _{n \rightarrow \infty}\left(\sum_{m=1}^{n} \frac{1}{m}-\ln n\right)
$$

but for this kind of sequence the Toader's Theorem, [3], is useless due to the presence of the logarithm. When we are in presence of sequences of logarithmic growth, the evaluation of the limit (2) is possibile using ad hoc techniques. In this note we use some series summation formulae given in [2] to evaluate some limits of the form (2) in presence of sequences with logarithmic growth.

\section{The limits}

Here we evaluate five limits of sequences in the form (2). All the sequences cannot be evaluated with the Toader's Theorem, but using five identities from $[2]$. 
Theorem 2.1. If $\zeta(s)$ denotes the Riemann's zeta function

$$
\zeta(s)=\sum_{n=1}^{\infty} \frac{1}{n^{s}}, \quad s>1
$$

then the following identities hold true:

$$
\begin{aligned}
& \lim _{n \rightarrow \infty}\left(\sum_{m=2}^{n} \frac{\zeta(m)}{m}-\ln n\right)=0 \\
& \lim _{n \rightarrow \infty}\left(\sum_{m=1}^{n} \frac{\zeta(2 m+1)}{m+1}-\ln n\right)=\ln 2-1 \\
& \lim _{n \rightarrow \infty}\left(\sum_{m=1}^{n} \frac{\zeta(2 m)}{m}-\ln (n)\right)=\gamma+\ln 2 \\
& \lim _{n \rightarrow \infty}\left(\sum_{m=2}^{n} \frac{\zeta(m)}{m+1}-\ln n\right)=\frac{1}{2}(\gamma-\ln (2 \pi)) \\
& \lim _{n \rightarrow \infty}\left(\sum_{m=1}^{n} \frac{\zeta(2 m)}{m+1}-\ln n\right)=\gamma-\ln \pi+\frac{1}{2}
\end{aligned}
$$

Proof. To show (4) we start form identity (158) p. 281 of [2]

$$
\sum_{n=2}^{\infty} \frac{\zeta(n)-1}{n}=1-\gamma
$$

where $\gamma$ is the Euler-Mascheroni constant defined in (3). Notice that, for $n \in \mathbb{N}, n>2$, we have

$$
\sum_{m=2}^{n} \frac{\zeta(n)-1}{n}=\sum_{m=2}^{n} \frac{\zeta(m)}{m}-\sum_{m=2}^{n} \frac{1}{m}
$$

On the other side from (3) we also get

$$
\sum_{m=2}^{n} \frac{1}{m}=\gamma-1+\ln n+\mathrm{o}(1)
$$

being o(1) a vanishing sequence as $n \rightarrow \infty$. Substituting in (10) we find

$$
\sum_{m=2}^{n} \frac{\zeta(n)-1}{n}=\sum_{m=2}^{n} \frac{\zeta(m)}{m}-\gamma+1-\ln n+\mathrm{o}(1)
$$

or

$$
\sum_{m=2}^{n} \frac{\zeta(n)-1}{n}+(\gamma-1)=\sum_{m=2}^{n} \frac{\zeta(m)}{m}-\ln n+\mathrm{o}(1)
$$


Taking the limit for $n \rightarrow \infty$ in (13), remembering (9) which implies that the left hand side of (13) vanishes we get formula (4).

The proof for the remaining four limits $(5),(6),(7)$ and (8) is similar. The relevant starting identities are, in order, equation 503 p. 314 of [2]:

$$
\sum_{n=1}^{\infty} \frac{\zeta(n+1)-1}{n+1}=\ln 2-\gamma,
$$

formula (159) p. 281 of [2]:

$$
\sum_{n=1}^{\infty} \frac{\zeta(2 n)-1}{n}=\ln 2
$$

formula 500 p. 314 di [2]:

$$
\sum_{n=2}^{\infty} \frac{\zeta(n)-1}{n+1}=\frac{3}{2}-\frac{\gamma}{2}-\ln \sqrt{2 \pi}
$$

and formula (545) p. 320 of [2]:

$$
\sum_{n=1}^{\infty} \frac{\zeta(2 n)-1}{n+1}=\frac{3}{2}-\ln \pi
$$

\section{Conclusion}

In this short note we presented the evaluation of five limits similar to the well known limit which leads to the well known Euler-Mascheroni $\gamma$ constant. The presence of the logarithm indicates that in any occurrence a particular technique is needed: we employed five identities established in [2]. All the limit, are for the best of our knowledge, new.

\section{References}

[1] J. Havil, Gamma. Exploring Euler's Constant, Princeton University Press, Princeton, 2003.

[2] H.M. Srivastava and J. Choi, Zeta and q-Zeta Functions and Associated Series and Integrals, Elsevier, Amsterdam, 2012

[3] G. Toader, Lalescu Sequences, Univ. Beograd. Publ. Elektrothn. Fak. Ser. Mat., 9 (1988), 25-25.

Received: September 1, 2014 